\title{
Dialisi e secchezza cutanea: riflessioni e rimedi
}

\author{
Stefania Motta \\ Università degli Studi di Milano e Humanitas Research Hospital, Milano
}

\begin{abstract}
Dialysis and dry skin: considerations and remedies
Uremic pruritus is the most distressing symptom of renal disease, even in patients adequately dialysed. Dry skin (xerosis) is the most frequent cutaneous manifestation of renal disease and has been suggested as a cause of uraemic pruritus. Despite a plethora of suggested mechanisms, the pathophysiology of uraemic pruritus remains elusive. Although a positive correlation between the severity of clinical dryness and pruritus has been shown, it has been impossible to find a relationship between trans-epidermal water loss, or skin hydration, and pruritus. Recent studies indicate that the reduction in glycerol content influences skin hydration and independently influences the epidermal barrier function. In order to counteract skin dryness it is recommended to correctly clean the body and to use an adequate topical product. A non foaming cleansing cream easy rinsing with mild tensioactive agents is recommended. Then a product able to attract water from inner skin and to retain it inside the stratum corneum, thus restoring its plasticity and smoothness, has to be applied. The application of an ointment with auto-hydrating activity containing a mixture of polymeric long chain vegetable polyglycerols combined with a blend of polyisobutenes provides a quick healing of xerosis.
\end{abstract}

Keywords: Dialysis, Uraemic pruritus, Dry skin, Xerosis, Epidermal barrier function, Itch, Body cleansing, Autohydrating ointment, Polyglycerols, Polyisobutenes

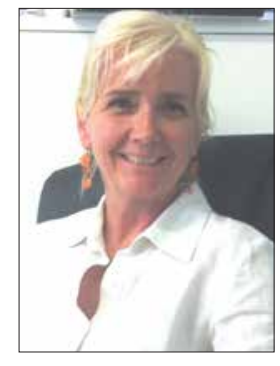

\section{Introduzione}

I pazienti con malattia cronica renale in dialisi sviluppano molti problemi cutanei come quelli riportati qui di seguito (1).

Manifestazione clinica

- Unghia metà bianca

Stefania Motta
- Prurito

- Xerosi

- Iperpigmentazioni

- Ecchimosi

- Ipercheratosi follicolare

- Dermatiti perforanti

- Calcinosi cutanea

- Calcifilassi

- Fibrosi sistemica nefrogenica

- Porfiria cutanea tarda

- Pseudoporfiria

Accepted: May 11, 2015

Published online: May 28, 2015

Indirizzo per la corrispondenza:

Stefania Motta

Università degli Studi di Milano e Humanitas

Research Hospital, Milano

stefania.motta@humanitas.it
I più comuni tra questi disturbi sono: il prurito uremico, la Xerosi e le iperpigmentazioni.

Sulla genesi di questi problemi e, di conseguenza, sui rimedi, si sa ancora troppo poco, e questo è dovuto alla scarsità di studi mirati sull'argomento.

In questo articolo viene presa in considerazione la problematica della Xerosi cutanea.

Nel contesto dell'emodialisi, la Xerosi cutanea è anche denominata Xerosi uremica o ittiosi acquisita.

I più recenti studi sull'argomento riportano che una percentuale che va dal $50 \%$ all' $85 \%$ dei pazienti in emodialisi soffre di Xerosi cutanea $(2,3)$.

La Xerosi può essere localizzata o generalizzata ed è di più frequente osservazione alle estremità, sia braccia/mani che gambe/piedi (4).

Lo stato di Xerosi cutanea, di per sé, è una causa di prurito che, nel caso del paziente dializzato, può andare a sovrapporsi al prurito uremico. Le due forme di prurito vanno, però, tenute separate, perché il prurito uremico può essere presente anche in assenza di manifestazioni di Xerosi cutanea.

A parte il prurito e il disagio, la Xerosi è anche responsabile delle infezioni cutanee e del ritardo di guarigione delle ferite (5).

\section{Manifestazioni cliniche}

La Xerosi cutanea è più evidente alle estremità, soprattutto su gambe, mani e piedi. Si va da una semplice accentuazione 


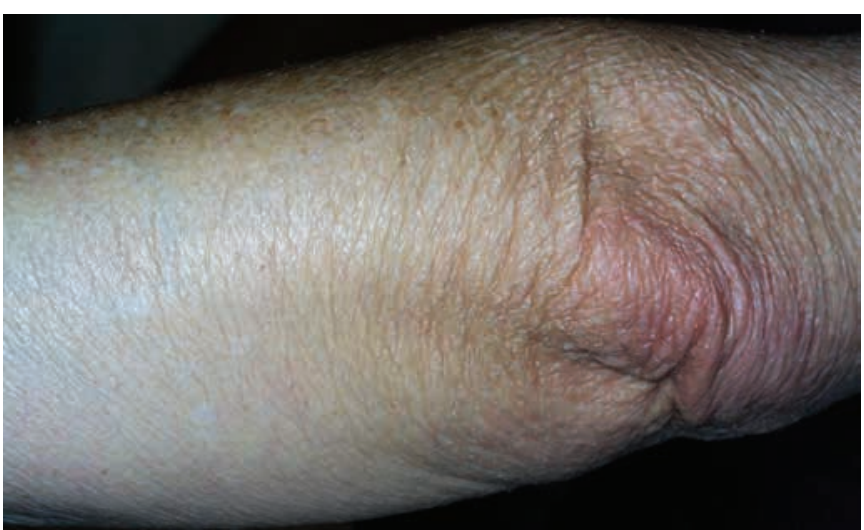

Fig. 1 - Xerosi cutanea iniziale al gomito: accentuazione della tramatura cutanea.

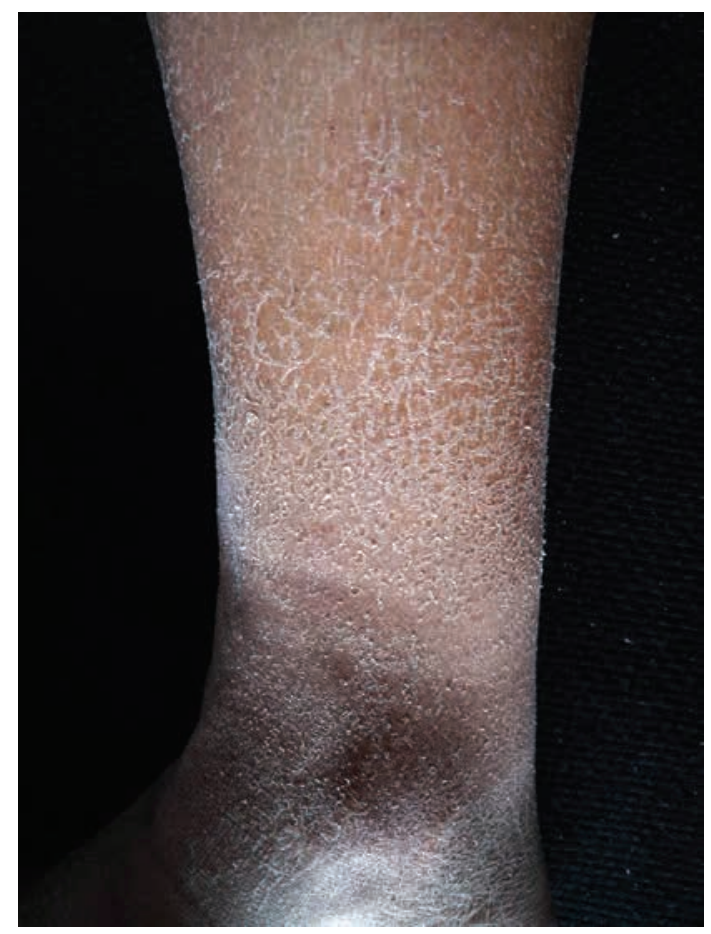

Fig. 2 - Xerosi cutanea alla gamba: comparsa di un reticolato bianco con desquamazione.

della tramatura cutanea (Fig. 1), alla comparsa di un reticolato bianco con desquamazione evidente (Fig. 2), mentre nei casi più gravi appare una desquamazione accompagnata da arrossamento cutaneo (Fig. 3) fino alla comparsa di fissurazioni dello strato corneo (Fig. 4). Il grado di secchezza può variare di molto e lo stesso vale per l'aspetto clinico.

\section{Patogenesi della Xerosi}

Lo strato corneo normale è dotato di notevoli proprietà viscoelastiche, così da assecondare le modificazioni cutanee imposte dalle contrazioni muscolari e dalle articolazioni.

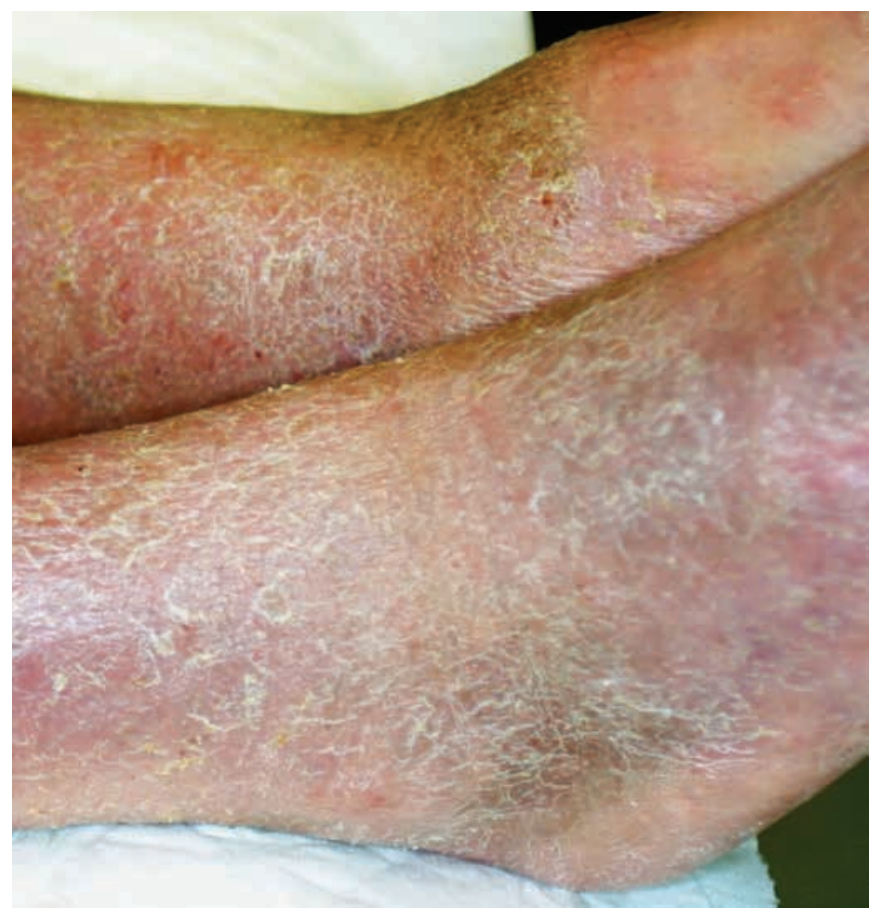

Fig. 3 - Xerosi cutanea del dorso del piede: desquamazione evidente con arrossamento.

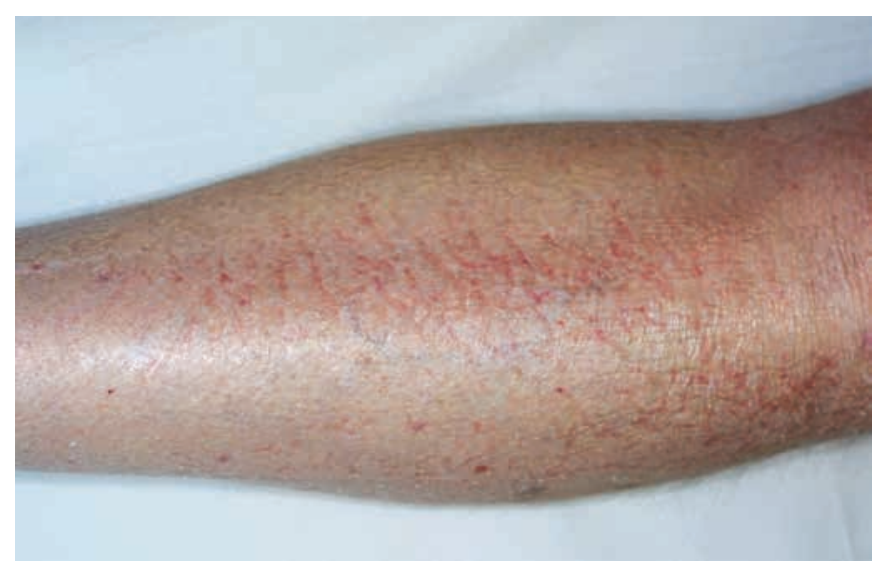

Fig. 4 - Xerosi cutanea al braccio: presenza di fissurazioni.

Queste proprietà sono dovute alla particolare composizione dello strato corneo e, in particolare, alla flessibilità delle lamelle cornee immerse nei lipidi interlamellari prodotti dai cheratinociti. L'insieme di lamelle cornee e di lipidi interlamellari compongono la Funzione Barriera Epidermi$\mathrm{ca}$, che difende l'organismo dalla penetrazione di agenti nocivi e che, nel contempo, previene la perdita di acqua verso l'ambiente.

La Xerosi cutanea è essenzialmente dovuta alla diminuzione del contenuto di acqua dello strato corneo.

La diminuzione dell'acqua dello strato corneo può, a sua volta, essere dovuta al danno della Funzione Barriera o al di- 


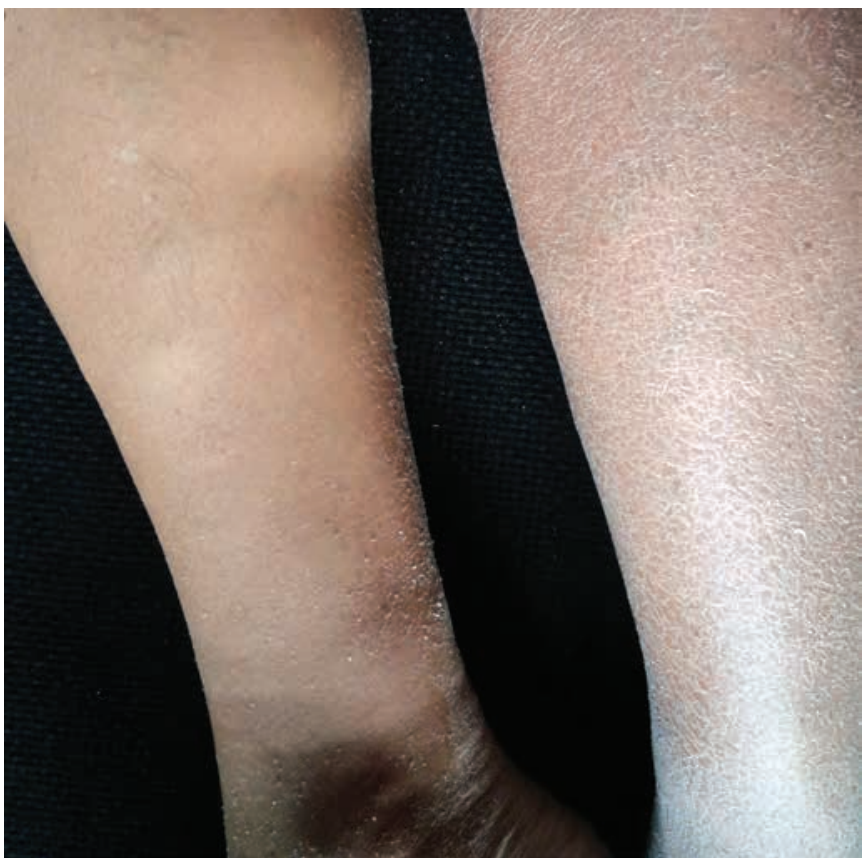

Fig. 5 - A sinistra - esempio di una gamba trattata con unguento auto-idratante.

fetto di sintesi dei leganti delle molecole d'acqua presenti sia sui lipidi sia sulle proteine che compongono lo strato corneo.

L'esempio del primo caso è dato dall'eccesso di lavaggi con detergenti, mentre quello del secondo è l'invecchiamento cutaneo.

La Xerosi del paziente dializzato è stata variamente interpretata. Per alcuni ricercatori sarebbe legata a una diminuzione di numero, dimensioni e funzioni delle ghiandole sudorali e sebacee (6).

Questa interpretazione convince poco perché sia le ghiandole sudorali che quelle sebacee contribuiscono poco o per nulla all'idratazione dello strato corneo.

Elias et al. hanno analizzato anche un eventuale difetto della Funzione Barriera nei pazienti dializzati, non trovando, però, una correlazione tra questo e la Xerosi (7). L'integrità dello strato corneo risulta sì alterata in quei pazienti che hanno prurito e che, grattandosi, danneggiano le lamelle cornee, ma, poi, il recupero dell'omeostasi è normale; nemmeno il valore del $\mathrm{pH}$ cutaneo leggermente superiore ai controlli risulta significativo.

È più probabile che la Xerosi sia dovuta a un difetto di sintesi dei leganti molecolari dell'acqua. Gli stessi Autori, infatti, hanno dimostrato un difetto del contenuto di glicerolo nello strato corneo legato anche alla riduzione di glicerolo endogeno. Anche la diminuzione e le differenze nei lipidi presenti nel siero possono infliuenzare la sintesi e la quantità dei grassi dello strato corneo, che hanno un ruolo centrale nella pelle secca e nella Funzione Barriera Epidermica (7).

\section{Rimedi per contrastare la Xerosi}

In generale, per contrastare la Xerosi cutanea, si agisce sia sui lavaggi sia sull'applicazione di prodotti idonei allo scopo. Entrambi i provvedimenti servono sia da prevenzione che da trattamento della Xerosi.

La detersione è un punto cruciale per il trattamento della secchezza cutanea; infatti, tutti i detergenti, saponi sia veri che sintetici liquidi o solidi, hanno la capacità di aggredire ed estrarre parte dei lipidi interlamellari che formano la Funzione Barriera, quindi promuovono la Xerosi.

L'impiego delle creme cosiddette idratanti o emollienti il più delle volte non cambia la situazione di secchezza. Infatti le creme sono delle emulsioni contenenti un emulsionante che ha un'azione tensioattiva e, quindi, si comportano come dei saponi nei confronti della Funzione Barriera. Anche l'aggiunta di sostanze come l'urea nella crema non è indicata, poiché, una volta applicata, la crema si rompe, l'acqua dell'emulsione evapora, i grassi si assorbono e l'urea rimane in superficie o, peggio, può anch'essa assorbirsi provocando irritazione e peggiorando il prurito.

Per riuscire a contrastare la secchezza patologica come la Xerosi uremica occorre utilizzare particolari prodotti, come i detergenti non schiumogeni e gli unguenti detti "unguenti auto-idratanti".

L'esperienza degli Autori è stata fatta, oltre che nella Xerosi uremica, in altre situazioni di Xerosi cutanea patologica come quella dei trapiantati d'organo, dei soggetti tumorali in trattamento con inibitori EGFR o delle Kinasi endocellulari e dei soggetti sottoposti a radio e a chemioterapia.

In questi pazienti si raccomanda la detersione con detergenti non schiumogeni a base di tensioattivi (taurati, sarcosinati) meno aggressivi per i lipidi cutanei e a rapido risciacquo. Il rapido risciacquo garantisce che non rimangano tracce di detergente sulla pelle come, invece, succede regolarmente con i comuni detergenti.

Al posto delle creme idratanti o emollienti si raccomanda l'unguento auto-idratante (Fig. 5).

Si tratta di un unguento composto da polimeri non assorbibili altamente idrofili. Una volta applicato, esso attira l'acqua dagli strati più profondi e, non essendo assorbibile, la trattiene nello strato corneo eliminando quasi istantaneamente lo stato di Xerosi.

\section{Disclosures}

Financial support: No financial support was received for this submission.

Conflict of interest: The author has no conflict of interest. 


\section{Bibliografia}

1. Markova A, Lester J, Wang J, Robinson-Bostom L. Diagnosis of common dermopathies in dialysis patients: a review and update. Semin Dial. 2012;25(4):408-18.

2. Udayakumar P, Balasubramanian S, Ramalingam KS, Lakshmi C, Srinivas CR, Mathew AC. Cutaneous manifestations in patients with chronic renal failure on hemodialysis. Indian J Dermatol Venereol Leprol. 2006;72(2):119-25.

3. Szepietowski JC, Balaskas E, Taube K, Taberly A, Dupuy P. Quality of life in patients with uraemic xerosis and pruritus. Acta Derm Venereol. 2011;91(3):313-7.

4. Khanna D, Singal A, Kalra OP. Comparison of cutaneous manifestations in chronic kidney disease with or without dialysis. Postgrad Med J. 2010;86(1021):641-7.

5. Avermaete A, Altmeyer P, Bacharach-Buhles M. Skin changes in dialysis patients: a review. Nephrol Dial Transplant. 2001;16 (12):2293-6.

6. Falodun O, Ogunbiyi A, Salako B, George AK. Skin changes in patients with chronic renal failure. Saudi J Kidney Dis Transpl. 2011;22(2):268-72.

7. Yosipovitch G, Duque MI, Patel TS, Elias PM. Skin barrier structure and function and their relationship to pruritus in end-stage renal disease. Nephrol Dial Transplant. 2007;22(11): 3268-72. 